\title{
The Asymptotic Expansions of Hankel Transforms and Related Integrals
}

\author{
By Robert F. MacKinnon
}

\begin{abstract}
In this paper, the asymptotic expansion of integrals of the form $\int_{0}^{\infty} F(k r) f(k) d k$ is considered, as $r$ tends to infinity, and where $F(k r)$ are Bessel functions of the first and second kind, or functions closely related to these. Asymptotic expansions for several functions of this type are presented under suitable restrictions on $f(k)$. The expansion given by Willis for Hankel transforms is seen to be valid under conditions of $f(k)$ less restrictive than those imposed by that author.
\end{abstract}

1. Introduction. The evaluation of the Hankel transform $\int_{0}^{\infty} J_{p}(k r) f(k) d k$ is a problem often arising in mathematical physics. So-called far-field approximations are of major interest and correspond to large values of the parameter $r$. A method for obtaining an asymptotic representation of the transform as a series in reciprocal powers of $r$ was given by Willis [1], who obtained as results the following:

$$
\int_{0}^{\infty} J_{0}(k r) f(k) d k=\frac{f(0)}{r}-\frac{1}{2} \frac{f^{\prime \prime}(0)}{r^{3}}+\frac{1}{2^{2}} \cdot \frac{3}{2 !} \frac{f^{\mathrm{iv}}(0)}{r^{5}}+\cdots
$$

and

$$
\int_{0}^{\infty} J_{1}(k r) f(k) d k=\frac{f(0)}{r}+\frac{f^{\prime}(0)}{r^{2}}-\frac{1}{2} \frac{f^{\prime \prime \prime}(0)}{r^{4}}+\cdots .
$$

where the primes denote differentiation with respect to $k$. As Willis pointed out, his restriction that $f(k)$ be regular analytic in the entire $k$-plane was too restrictive, since it could be shown that (1.1) remained valid for the function $(1+k)^{-1}$, as well as for a variety of functions of the type prescribed.

The Bessel function $J_{\nu}(k r)$ is oscillatory, so it is natural that consideration be given to the results available concerning the asymptotic representations of Fourier integrals. Several results given by Erdélyi [2] are used below, in particular:

THEOREM A. If $(\alpha, \beta)$ is a finite interval, and $\phi(k)$ is $N$ times continuously differentiable for $\alpha \leqq k \leqq \beta$, then

$$
\int_{\alpha}^{\beta} e^{i k r} \phi(k) d k=B_{N}(r)-A_{N}(r)+o\left(r^{-N}\right),
$$

where

$$
A_{N}(r)=\sum_{n=0}^{N-1} i^{n-1} \phi^{(n)}(\alpha) r^{-n-1} e^{i r \alpha}
$$

Received July 1, 1971.

AMS 1969 subject classifications. Primary 4430, 4433, 6555; Secondary 6520, 6525.

Key words and phrases. Asymptotic expansion, far-field approximation, integral transforms, Hankel transforms, Bessel functions, cylindrical functions, sine integral, Fresnel integral.

Copyright () 1972, American Mathematical Society 
and

$$
B_{N}(r)=\sum_{n=0}^{N-1} i^{n-1} \phi^{(n)}(\beta) r^{-n-1} e^{i r \beta} .
$$

THEOREM B. If $\phi(k)$ is $N$ times continuously differentiable, for $k \geqq \alpha, \phi^{(n)}(k) \rightarrow 0$ as $k \rightarrow \infty, n=0,1,2, \cdots, N-1$, and $\phi^{(N)}(k)$ is integrable over $(\alpha, \infty)$, then

$$
\int_{\alpha}^{\infty} e^{i k r} \phi(k) d k=-A_{N}(r)+o\left(r^{-N}\right) .
$$

In order to apply these theorems to Hankel transforms, it is convenient to assume that all functions $f(k)$ are continuously differentiable except possibly at a finite number of points. Thus, the range of integration may be divided into intervals over which $f(k)$ is continuously differentiable except possibly at endpoints in which case integrals are defined through imposition of appropriate limits.

The above theorems can be used to obtain corresponding results for the kernels $\cos k r$ and $\sin k r$. The exact forms these results take are not essential to the analysis below. The relevant property of the expansions is that the terms $A_{N}(r)$ and $B_{N}(r)$ are linear in $\phi$ and its derivatives evaluated at the endpoints; and this property will be maintained for the trigonometric kernels. It is assumed implicitly that $\phi(k) e^{i k r}$ does not possess points of stationary phase in the range of integration, since their presence entails contributions at points other than $\alpha$ and $\beta$.

2. Relationships to Fourier Integrals. For $k r>0, J_{\nu}(k r)$ may be expressed in the form (Watson [3])

$$
J_{,}(k r)=(2 / \pi k r)^{1 / 2}[\cos \chi P(k r, \nu)-\sin \chi Q(k r, \nu)],
$$

where

$$
\begin{gathered}
\chi=k r-\frac{1}{2} \nu \pi-\frac{1}{2} \pi, \\
P(k r, \nu)=\sum_{m=0}^{M}(-)^{m}(\nu, 2 m)(2 k r)^{-2 m}+o\left(r^{-2 M}\right),
\end{gathered}
$$

and

$$
Q(k r, \nu)=\sum_{m=0}^{M-1}(-)^{m}(\nu, 2 m+1)(2 k r)^{-2 m-1}+o\left(r^{-2 M}\right) .
$$

We define the function $J(k r, \nu, M)$ such that

$$
J(k r, \nu, M)=(2 / \pi k r)^{1 / 2}[\cos \chi \hat{P}(k r, \nu)-\sin \chi \hat{Q}(k r, \nu)],
$$

where $\hat{P}$ and $\hat{Q}$ are the finite sums appearing in (2.3) and (2.4), respectively. Now,

$$
\begin{aligned}
\int_{\alpha}^{\beta} J(k r, \nu, M) f(k) d k=\sum_{m=0}^{2 M} & a_{m} r^{-m-1 / 2} \int_{\alpha}^{\beta} \cos k r f(k) k^{-m-1 / 2} d k \\
& +b_{m} r^{-m-1 / 2} \int_{\alpha}^{\beta} \sin k r f(k) k^{-m-1 / 2} d k
\end{aligned}
$$

where $a_{m}$ and $b_{m}$ are linear combinations of the coefficients of the terms in $\hat{P}$ and $\hat{Q}$.

Theorems $\mathrm{A}$ and $\mathrm{B}$ may be applied to the integrals appearing in (2.6) to yield 
expansions of $o\left(r^{-N-1 / 2}\right)$ where, by choice, $2 M>N$. The expansion of each integral for a given $m$ is a sum of terms linear in $f(k)$ and its $N-1$ derivatives, the number of terms being dependent on $m$ and $N$. The resulting asymptotic approximation is linear in $a_{m}, b_{m}$ and in $f(k)$ and its derivatives.

Theorems A and B impose the requirement that $\phi(k)$ be $N$ times continuously differentiable in $(\alpha, \beta)$. Theorem B requires that $\phi^{(n)}(k) \rightarrow 0$ as $k \rightarrow \infty$ for $0 \leqq n \leqq$ $N-1$ and that $\phi^{(N)}(k)$ be integrable over $(\alpha, \infty)$. If $f(k)$ possesses these properties, so does $f(k) k^{-m-1 / 2}$ for $m \geqq 0$ and $\alpha>0$.

Since, for some finite value $F(M, \nu)$,

$$
\left|J_{\nu}(k r)-J(k r, \nu, M)\right|<F(M, \nu) k^{-2 M-1 / 2} r^{-2 M-1 / 2},
$$

it follows that

$$
\left|\int_{\alpha}^{\beta}\left\{J_{\nu}(k r)-J(k r, \nu, M)\right\} f(k) d k\right|<F(M, \nu) r^{-2 M-1 / 2} \int_{\alpha}^{\beta}|f(k)| k^{-2 M-1 / 2} d k,
$$

hence, if $|f(k)| k^{-2 M-1 / 2}$ is integrable,

$$
\int_{\alpha}^{\beta} J_{\nu}(k r) f(k) d k=\int_{\alpha}^{\beta} J(k r, \nu, M) f(k) d k+o\left(r^{-2 M}\right) .
$$

Thus, the following propositions may be stated.

LEMMA 1. If $(\alpha, \beta)$ is a finite interval and $f(k)$ is $N$ times continuously differentiable for $0<\alpha \leqq k \leqq \beta$, the asymptotic approximation of $\int_{\alpha}^{\beta} J_{\nu}(k r) f(k) d k$ to $o\left(r^{-N-1 / 2}\right)$ equals the asymptotic approximation of $\int_{\alpha}^{\beta} J(k r, \nu, M) f(k) d k$ to $o\left(r^{-N-1 / 2}\right)$, provided that $2 M>N$.

LEMMA 2. If $f(k)$ is $N$ times continuously differentiable for $k \geqq \alpha>0, f^{(n)}(k) \rightarrow 0$ as $k \rightarrow \infty, 0 \leqq n \leqq N-1$, and $f^{(N)}(k)$ is integrable over $(\alpha, \infty)$, then the asymptotic approximation of $\int_{\alpha}^{\infty} J_{\nu}(k r) f(k) d k$ to $o\left(r^{-N-1 / 2}\right)$ equals the asymptotic approximation of $\int_{\alpha}^{\infty} J(k r, \nu, M) f(k) d k$ to $o\left(r^{-N-1 / 2}\right)$, provided that $2 M>N$.

If $\phi(k)$ is infinitely differentiable in $(\alpha, \beta)$, Theorems A and B imply that asymptotic expansions may be formed by allowing $N$ to tend to infinity, that is to say, $A_{N}$ and $B_{N}$ become infinite series (denoted as $A_{\infty}$ and $B_{\infty}$ ) the remainders for which are less in order than any negative power of $r$. For this situation, there exists an asymptotic expansion for $\int_{\alpha}^{\beta} J(k r, \nu, M) f(k) d k, M$ finite, in which appear derivatives of $f(k)$. to all orders. The asymptotic approximation of this integral to $o\left(r^{-N-1 / 2}\right)$ consists of a sum of terms, finite in number, which are $o\left(r^{-N-1 / 2}\right)$ or less. These observations lead to the following lemma.

LEMMA 3. If $(\alpha, \beta)$ is a finite interval and $f(k)$ is infinitely differentiable for $0<\alpha \leqq k \leqq \beta$, then the asymptotic approximation of $\int_{\alpha}^{\beta} J_{\nu}(k r) f(k) d k$ to $o\left(r^{-N-1 / 2}\right)$ equals a partial sum of the asymptotic expansion of $\int_{\alpha}^{\beta} J(k r, \nu, M) f(k) d k$, provided that $2 M>N$.

3. Integrals with a Neutralizer. We introduce now a van der Corput neutralizer, $\eta(k)$, defined as follows:

$$
\eta(k)=q^{-1}(\alpha, \beta) \int_{k}^{\beta} e^{-(u-\alpha)} e^{-1 /(u-\alpha)-1 /(\beta-u)} d u,
$$

where 


$$
q(\alpha, \beta)=\int_{\alpha}^{\beta} e^{-(u-\alpha)} e^{-1 /(u-\alpha)-1 /(\beta-u)} d u .
$$

This neutralizer has the properties that

1. it is unity at $k=\alpha$;

2. it is finite and infinitely differentiable over $(\alpha, \beta)$;

3. its value at $\beta$ and the values of all its derivatives at $\alpha$ and $\beta$ are zero;

4. it retains the properties $1-3$ as $\beta \rightarrow \infty$.

Consider now the asymptotic expansion of $\int_{\alpha}^{\beta} \xi_{n}(k) J(k r, \nu, M) d k$ where $\xi_{n}(k)$ denotes $\left(\alpha^{n} / n !\right)(k / \alpha-1)^{n} \eta(k)$. As a result of properties 2 and 3, Theorem $\mathbf{A}$ may be applied to (2.6) to yield

$$
\begin{aligned}
\int_{\alpha}^{\beta} \xi_{n}(k) J(k r, \nu, M) d k \sim \sum_{m=0}^{2 M} & a_{m} r^{-m-1 / 2} U_{N}(r ; n ; m) \\
& \quad+b_{m} r^{-m-1 / 2} V_{N}(r ; n ; m)+o\left(r^{-N-1 / 2}\right),
\end{aligned}
$$

where $U_{N}$ and $V_{N}$ are linear combinations of $A_{N}$ defined by (1.4) for $\pm i$ with $\phi(k)$ taken to be $k^{-m-1 / 2} \xi_{n}(k)$. Except for the $n$th derivative whose value is unity, the derivatives of $\xi_{n}(k)$ are zero at $\alpha$; thus, $U_{N}$ and $V_{N}$ each consist of a sum of a finite number of nonzero terms, those terms which arise in the approximation to $o\left(r^{-N-1 / 2}\right)$ as factors of the $n$th derivative at $\alpha$. In other words, $\xi_{n}(k)$ serves to isolate the factors of the $n$th derivative at $\alpha$. In the extension of (2.6) for an infinitely differentiable $f(k), U_{N}$ and $V_{N}$ appear as factors of $f^{(n)}(\alpha)$. Thus, because of the linearity in $f^{(n)}(\alpha)$ in the expansions of Fourier integrals,

$$
\begin{aligned}
\int_{\alpha}^{\beta} J(k r, \nu, M) f(k) d k \sim & \sum_{m=0}^{2 M} a_{m} r^{-m-1 / 2} \sum_{n=0}^{\infty} f^{(n)}(\alpha) U_{N}(r ; n ; m) \\
& +b_{m} r^{-m-1 / 2} \sum_{n=0}^{\infty} f^{(n)}(\alpha) V_{N}(r ; n ; m) \\
& + \text { terms at } \beta+o\left(r^{-N-1 / 2}\right) \\
& \sim \sum_{n=0}^{\infty} f^{(n)}(\alpha) \sum_{m=0}^{2 M} a_{m} r^{-m-1 / 2} U_{N}(r ; n ; m) \\
& +b_{m} r^{-m-1 / 2} V_{N}(r ; n ; m)+\text { terms at } \beta+o\left(r^{-N-1 / 2}\right) .
\end{aligned}
$$

It is to be noted that for a given $N, U_{N}(r ; n ; m)$ and $V_{N}(r ; n ; m)$ are zero for $n>$ $N-m-1$, since terms arising under this condition are $o\left(r^{-N-1 / 2}\right)$. Since $\xi_{n}(k)$ is infinitely differentiable, $N$ may be taken arbitrarily large so that (3.4) is seen to be an asymptotic expansion for an infinitely differentiable $f(k)$. These observations lead to the following lemma.

LEMMA 4. If $(\alpha, \beta)$ is a finite interval and $f(k)$ is infinitely differentiable for $0<$ $\alpha \leqq k \leqq \beta$, the asymptotic approximation of $\int_{\alpha}^{\beta} J_{\nu}(k r) f(k) d k$ to o $\left(r^{-N-1 / 2}\right)$ contains terms cvaluated at $\alpha$ which are those terms to $o\left(r^{-N-1 / 2}\right)$ in the asymptotic expansion,

$$
\sum_{n=0}^{\infty} \frac{\alpha^{n} f^{(n)}(\alpha)}{n !} \int_{\alpha}^{\beta}\left(\frac{k}{\alpha}-1\right)^{n} J_{\nu}(k r) \eta(k) d k .
$$

If the most that can be said of $f(k)$ is that it is $N$ times continuously differentiable, the sum proceeds over $n$ from 0 to $N-1$. 
Similar lemmas can be derived for the terms at $\beta$ and for infinite intervals of integration. In particular, for the terms at $\beta$, the following lemma holds.

Lemma 5. Under the conditions of Lemma 4, the terms to $o\left(r^{-N-1 / 2}\right)$, evaluated at $\beta$, are the terms to $o\left(r^{-N-1 / 2}\right)$ in the asymptotic expansion of

$$
\sum_{n=0}^{\infty} \frac{\beta^{n} f^{(n)}(\beta)}{n !} \int_{\alpha}^{\beta} J_{\nu}(k r)\left(\frac{k}{\beta}-1\right)^{n}(1-\eta(k)) d k .
$$

4. Integrals for $k<\alpha$. It is to be expected that, as $r$ tends to infinity, the behaviour of $f(k)$ near the origin becomes critical in the determination of the transform. Suppose $f(k)$ is expandable in a Taylor series in the interval $-\epsilon_{1}<k<2 \alpha+\epsilon_{2}$, with $\alpha>0$ and $\epsilon_{1,2}>0$. Since a Taylor series is uniformly convergent within its radius of convergence, it follows that

$$
\begin{aligned}
\int_{0}^{\alpha} J_{\nu}(k r) f(k) d k & =\sum_{n=0}^{\infty} \frac{\alpha^{n} f^{(n)}(\alpha)}{n !} \int_{0}^{\alpha}\left(\frac{k}{\alpha}-1\right)^{n} J_{\nu}(k r) d k, \\
& =\sum_{n=0}^{\infty} \frac{f^{(n)}(0)}{n !} \int_{0}^{\alpha} J_{\nu}(k r) k^{n} d k .
\end{aligned}
$$

Let the functions $G(x, n, \nu)$ and $C(n, \nu)$ be defined by

$$
\int_{0}^{x} u^{n} J_{\nu}(u) d u=C(n, \nu)+G(x, n, v) .
$$

Properties of $C$ and $G$ are given by Luke [4]; in particular,

$$
C(n, \nu)=2^{n} \Gamma\left(\frac{\nu+n+1}{2}\right) / \Gamma\left(\frac{\nu-n+1}{2}\right)
$$

and, as $x$ tends to infinity,

$$
G(x, n, v) \sim(2 / \pi x)^{1 / 2} x^{n}(h \cos x+g \sin x),
$$

where $h$ and $g$ possess asymptotic power series expansions in $x$. Equations (4.4) and (4.5) are valid if $R(n+\nu)>-1$. It is shown below that the $C(n, \nu)$ appear as coefficients in the asymptotic approximation of Hankel transforms.

5. Approximation to Hankel Transforms. The results given above can be used to derive expansions for integrals of $J_{v}(k r) f(k)$ under various conditions on $f(k)$ and on the intervals of integration, provided that $f(k)$ is continuously differentiable at all but a finite number of points in the interval. Suppose an interval $(\alpha, \gamma)$, finite or infinite, with $\alpha>0$, is divided into two regions $(\alpha, \beta)$ and $(\beta, \gamma)$ such that $f(k)$ is $N$ times continuously differentiable over $(\alpha, \beta)$ and $N^{\prime}$ times continuously differentiable over $(\beta, \gamma)$ with $N^{\prime}>N$. Further, suppose that $f^{(n)}(\beta)$ is continuous at $\beta$ for $n=0,1,2, \cdots, j \leqq N$. Under these conditions, the first $j$ terms, evaluated at $f$ in the expansions for $(\alpha, \beta)$ and for $(\beta, \gamma)$, cancel. This can be seen from an application of Theorem A and/or B to integrals of $J(k r, \nu, M) f(k), M>\frac{1}{2} N$. The approximation to $o\left(r^{-i-1 / 2}\right)$ of the integral over $(\alpha, \gamma)$ will not contain terms evaluated at $\beta$, which is to be expected since $f(k)$ is $j$ times continuously differentiable in $(\alpha, \gamma)$. An approximation to $o\left(r^{-p-1 / 2}\right)$, with $N<p \leqq N^{\prime}$, would contain terms evaluated at $\beta$ in the limits taken from above and from below. For generality of 
proof, it is not necessary to consider more than one subdivision of the interval $(0, \infty)$.

A case of practical interest is dealt with in the following theorem.

THEOREM 1. The asymptotic approximation of the Hankel transform of a function $f(k)$ may be expressed in the form

$$
\begin{aligned}
\int_{0}^{\infty} J_{\nu}(k r) f(k) d k= & \sum_{n=0}^{N-1} \frac{f^{(n)}(0)}{n !} \frac{1}{r^{n+1}} C(n, \nu)+o\left(r^{-N-1 / 2}\right), \\
& \text { where } C(n, \nu)=2^{n} \Gamma\left(\frac{\nu+n+1}{2}\right) / \Gamma\left(\frac{\nu-n+1}{2}\right),
\end{aligned}
$$

provided that

(1) $R(\nu)>-1$;

(2) $f(k)$ is regular analytic in a region of the complex $k$-plane which includes an interoal $-\epsilon_{1} \leqq k \leqq \beta+\epsilon_{2}$, for $\epsilon_{1}, \epsilon_{2}$ and $\beta>0$;

(3) $f(k)$ is $N$ times continuously differentiable for $k>0$;

(4) as $r \rightarrow \infty, e^{ \pm i k r} f(k)$ have no stationary points for $k>\beta / 2$;

(5) $k^{-1 / 2} f^{(n)}(k) \rightarrow 0$ as $k \rightarrow \infty, n=0,1, \cdots, N-1$; and

(6) $k^{-1 / 2} f^{(N)}(k)$ is integrable over $(\beta, \infty)$.

Proof. Choose $\alpha$ so that $0<\alpha<\left(\beta+\epsilon_{2}\right) / 2$ and there are no stationary points in $(\alpha, \beta)$ for $e^{ \pm i k r} f(k)$. Consider the intervals of integration $(0, \alpha),(\alpha, \beta)$ and $(\beta, \infty)$. The asymptotic approximation of the integral over $(0, \beta)$ may be expressed as a sum of the expansion of the integral from $(0, \alpha)$ given by $(4.1)$, the terms to $o\left(r^{-N-1 / 2}\right)$ in the expansion given in Lemma 4 and the terms to $o\left(r^{-N-1 / 2}\right)$ evaluated at $\beta$. The terms at $\alpha$ to $o\left(r^{-N-1 / 2}\right)$ for $(\alpha, \beta)$ plus the expansion are the terms to $o\left(r^{-N-1 / 2}\right)$ of the sum

$$
\begin{aligned}
\sum_{n=0}^{\infty} \frac{f^{(n)}(\alpha)}{n !} \alpha^{n} & \int_{0}^{\alpha}\left(\frac{k}{\alpha}-1\right)^{n} J_{\nu}(k r) d k \\
& +\sum_{n=0}^{\infty} \frac{f^{(n)}(\alpha)}{n !} \alpha^{n} \int_{\alpha}^{\beta}\left(\frac{k}{\alpha}-1\right)^{n} \eta(k) J_{\nu}(k r) d k \\
& =\sum_{n=0}^{\infty} \frac{f^{(n)}(\alpha)}{n !} \alpha^{n} \int_{0}^{\beta}\left(\frac{k}{\alpha}-1\right)^{n} H(k) J_{\nu}(k r) d k \\
& =\sum_{n=0}^{\infty} \frac{f^{(n)}(0)}{n !} \int_{0}^{\beta} J_{\nu}(k r) k^{n} H(k) d k,
\end{aligned}
$$

where

$$
\begin{aligned}
H(k) & =\eta(k), & & \text { for } \alpha \leqq k \leqq \beta, \\
& =1, & & \text { for } 0 \leqq k \leqq \alpha .
\end{aligned}
$$

The function $H(k)$ and all its derivatives are continuous at $k=\beta$.

Now, from integration by parts,

$$
\int_{0}^{\beta} J_{\nu}(k r) k^{n} H(k) d k=r^{-n-1} C(n, \nu)-r^{-n} \int_{\alpha}^{\beta} H^{\prime}(k) G(k r, n, \nu) d k,
$$

where the prime denotes differentiation with respect to $k$. The function $G(k r, n, \nu)$ is of the same form as $J_{,}(k r)$, in the sense that it possesses an asymptotic expansion 
in negative powers of $k r$ with cofactors $\sin k r$ and $\cos k r$. The conditions leading to Lemma 3 are satisfied by $G(k r, n, \nu)$ and its asymptotic approximation to $o\left(r^{-\mu-1 / 2}\right)$, $M>N$; hence, since $H^{\prime}(k)$ and all its derivatives are zero at $\alpha$ and at $\beta$, and since there are no stationary points in the interval $(\alpha, \beta)$,

$$
\int_{0}^{\beta} J_{\nu}(k r) k^{n} H(k) d k=r^{-n-1} C(n, \nu)+o\left(r^{-M-1 / 2}\right),
$$

where $M>N$.

Now, from Lemmas 1 and 2 and from the forms of $A_{n}(r)$ and $B_{n}(r)$ given in (1.4) and (1.5), it follows that the terms at $\beta$ to $o\left(r^{-N-1 / 2}\right)$ arising from the asymptotic approximation to the integral over $(\alpha, \beta)$ cancel the terms at $\beta$ to $o\left(r^{-N-1 / 2}\right)$ arising from the asymptotic approximation to the integral over $(\beta, \infty)$. There are no terms arising at infinity or from stationary points. Hence, the only terms remaining are those to $o\left(r^{-N-1 / 2}\right)$ in the expansion

$$
\begin{aligned}
\sum_{n=0}^{\infty} \frac{f^{(n)}(0)}{n !} \int_{0}^{\beta} J_{\nu}(k r) k^{n} H(k) d k & =\sum_{n=0}^{\infty} \frac{f^{(n)}(0)}{n !} \frac{C(n, \nu)}{r^{n+1}} \\
& =\sum_{n=0}^{N-1} \frac{f^{(n)}(0)}{n !} \frac{C(n, \nu)}{r^{n+1}}+o\left(r^{-N-1 / 2}\right) .
\end{aligned}
$$

The theorem follows immediately. Q.E.D.

COROLLARY. Under the conditions of the theorem, the asymptotic approximation of the Hankel transform may be expressed in the form

$$
\int_{0}^{\infty} J_{v}(k r) f(k) d k=\sum_{n=0}^{N-1}(-)^{n} f^{(n)}(0) \gamma(n, r, v)+o\left(r^{-N-1 / 2}\right),
$$

where $\gamma(n, r, \nu)$ is the nth term in the asymptotic power series expansion of the transform of $e^{-k}$.

This follows immediately from an application of the theorem to $e^{-k}$. In essence, this corollary is a confirmation of the observation of Willis [1] for Hankel transforms under less restrictive conditions of $f(k)$. For example, the theorem and its corollary apply to $(\epsilon+k)^{-1}$ for $\epsilon>0$.

Consider a function $k^{v} f(k)$, where $f(k)$ satisfies the conditions for the validity of (5.1) and $\nu$ is not an integer. Because of its branch point at the origin, this function does not satisfy the conditions of (5.1). Since $k^{\nu} J_{v}(k r) f(k)$ is well-behaved near $k=0$, it is to be expected that the above conditions are too restrictive with regard to behaviour near the origin. It is well known (Luke [4]) that

$$
\int_{0}^{x} u^{\mu+n} J_{\nu}(u) d u=D(n, \nu, \mu)+E(k r, n, \nu, \mu),
$$

where

$$
D(n, \nu, \mu)=2^{\mu+n} \Gamma\left(\frac{\nu+n+\mu+1}{2}\right) / \Gamma\left(\frac{\nu-\mu-n+1}{2}\right) .
$$

and

$$
E(k r, n, \nu, \mu) \sim(2 \pi / k r)^{1 / 2}(k r)^{\mu+n}(h \cos \chi+g \sin \chi) .
$$


where $h$ and $g$ possess asymptotic power series expansions in the reciprocal of $k r$, under the condition that $R(\mu+\nu+n)>-1$. The arguments leading to the theorems given above, which rely on properties of $C(n, \nu)$ and $G(k r, n, \nu)$, apply equally well to $D$ and $E$. Thus, extensions of these theorems are readily obtainable as follows.

THEOREM 2. The asymptotic approximation of the Hankel transform of a function $k^{\mu} f(k)$ may be expressed in the form

$$
\begin{aligned}
& \int_{0}^{\infty} J_{,}(k r) k^{\mu} f(k) d k=\sum_{n=0}^{m} \frac{f^{(n)}(0)}{n !} \frac{1}{r^{n+1+\mu}} D(n, \nu, \mu)+o\left(r^{-N-1 / 2-R(\mu)}\right), \\
& \text { where } D(n, \nu, \mu)=2^{\mu+n} \Gamma\left(\frac{\nu+\mu+n+1}{2}\right) / \Gamma\left(\frac{\nu-\mu-n+1}{2}\right),
\end{aligned}
$$

and $m$ is chosen such that $m+2+R(\mu)>N+\frac{1}{2} \geqq m+1+R(\mu)$, provided that

(1) $R(\mu+\nu)>-1$;

(2) $f(k)$ is regular analytic in a region of the complex $k$-plane which includes an interval $-\epsilon_{1} \leqq k \leqq \beta+\epsilon_{2}$, for $\epsilon_{1}, \epsilon_{2}$ and $\beta>0$;

(3) $f(k)$ is $N$ times continuously differentiable for $k>0$;

(4) as $r \rightarrow \infty, e^{ \pm i k r} f(k) k^{\mu}$ have no stationary points for $k>\beta / 2$; and

(5) the nth derivative of $k^{\mu-1 / 2} f(k)$ tends to zero as $k \rightarrow \infty, n=0,1, \cdots, N-1$;

(6) the Nth derivative of $k^{\mu-1 / 2} f(k)$ is integrable over $(\beta, \infty)$.

Proof. As before, choose $\alpha$ such that $0<\alpha<\frac{1}{2}\left(\beta+\epsilon_{2}\right)$ and consider the intervals $(0, \alpha),(\alpha, \beta)$ and $(\beta, \infty)$. The terms in the asymptotic expansion to $o\left(r^{-m-1 / 2-R(\mu)}\right)$ of the integral over $(\alpha, \beta)$ must be those terms to $o\left(r^{-m-1 / 2-R(\mu)}\right)$ in the asymptotic expansion

$$
\sum_{n=0}^{\infty} \frac{\alpha^{n} f^{(n)}(\alpha)}{n !} \int_{\alpha}^{\beta}\left(\frac{k}{\alpha}-1\right)^{n} k^{\mu} J_{\nu}(k r) \eta(k) d k
$$

Adding to these terms the integral from $(0, \alpha)$, one finds terms of the form

$$
\sum_{n=0}^{\infty} \frac{f^{(n)}(0)}{n !} \int_{0}^{\beta} J,(\beta r) k^{n+\mu} H(k) d k,
$$

where $H(k)$ is defined as above. Now, provided $R(\mu+\nu)>-1$,

$$
\begin{aligned}
\int_{0}^{\beta} J_{\nu}(k r) k^{n+\mu} H(k) d k & \\
= & r^{-\mu-n-1} D(n, \nu, \mu)+r^{-\mu-n} \int_{0}^{\beta} H^{\prime}(k) E(k r, n, \nu, \mu) d k \\
= & r^{-\mu-n-1} D(n, \nu, \mu)+o\left(r^{-M-1 / 2}\right),
\end{aligned}
$$

where $M$ is chosen greater than $m+R(\mu)$.

From applications of Theorems A and B and Lemmas 1 and 2, it can be shown that the terms evaluated at $\beta$ for $(\beta, \infty)$ cancel terms at $\beta$ for $(\alpha, \beta)$ to $o\left(r^{-N-1 / 2-R(\mu)}\right)$. Hence, the asymptotic approximation of (5.12) follows immediately. Q.E.D.

Through use of Lemma 5, the finite Hankel transform can be treated similarly, but the results do not appear to be satisfactory. To the terms in (5.13) must be added terms from $\beta$, the upper limit. For $\mu=0$, these terms are terms from the expression 


$$
\begin{aligned}
\sum_{n=0}^{\infty} \frac{f^{(n)}(\beta)}{n !} & \sum_{m=0}^{n}(-)^{n-m}\left(\begin{array}{l}
n \\
m
\end{array}\right) \beta^{n-m} r^{-m-1} G(\beta r, m, \nu) \\
& =\sum_{n=0}^{\infty}\left\{\sum_{m=n}^{\infty} \frac{(-\beta)^{n-m} f^{(m)}(\beta)}{(m-n) !}\right\} r^{-n-1} \frac{G(\beta r, n, \nu)}{n !} .
\end{aligned}
$$

Since $r^{-n-1} G(\beta r, n, \nu)$ does not decrease in order of $r$ as $n$ increases, these expressions are not generally convenient for purposes of numerical evaluation.

6. Approximations for Related Integrals. The arguments leading to Theorem 2 may be applied immediately to integrals of $Y_{,}(k r)$, the result being the following theorem.

THEOREM 3. The asymptotic approximation to the $Y$-transform of $k^{\mu} f(k)$ may be expressed in the form

$$
\int_{0}^{\infty} Y_{\nu}(k r) k^{\mu} f(k) d k=\sum_{n=0}^{m} \frac{f^{(n)}(0)}{n !} r^{-n-1-\mu} K(n, \nu, \mu)+o\left(r^{-N-1 / 2-R(\mu)}\right),
$$

where $K(n, \nu, \mu)=\frac{2^{\mu+n}}{\pi} \Gamma\left(\frac{\mu+n+\nu+1}{2}\right) \Gamma\left(\frac{\mu+n-\nu+1}{2}\right) \sin (\mu+n-\nu) \pi / 2$, and $m$ is chosen such that $m+2+R(\mu)>N+\frac{1}{2} \geqq m+1+R(\mu)$, provided that

(1) $R(\mu \pm \nu)>-1$;

(2) $f(k)$ satisfies conditions (2)-(6) of Theorem 2.

Consideration of oscillatory factors of the form $\exp \left(i k^{\mu} r^{\mu}\right), \mu \geqq 1$, leads to a number of useful results for widely used functions of mathematical physics. The following is a particular case for a more general result of Erdélyi [2].

THEOREM C. If $\phi(k)$ is $N$ times continuously differentiable for $\alpha \leqq k \leqq \beta$, then, for $\mu \geqq 1$,

$$
\int_{\alpha}^{\beta} \phi(k) \exp \left(i r^{\mu} k^{\mu}\right) d k=B_{N}\left(r^{\mu}\right)-A_{N}\left(r^{\mu}\right)+o\left(r^{-\mu N}\right)
$$

where

$$
\begin{aligned}
& A_{N}\left(r^{\mu}\right)=-\sum_{n=0}^{N-1} g^{(n)}(\alpha) e^{i \pi(n+1) / 2} r^{-\mu(n+1)} \exp \left(i r^{\mu} \alpha^{\mu}\right) \\
& B_{N}\left(r^{\mu}\right)=-\sum_{n=0}^{N-1} g^{(n)}(\beta) e^{i \pi(n+1) / 2} r^{-\mu(n+1)} \exp \left(i r^{\mu} \beta^{\mu}\right)
\end{aligned}
$$

and where

$$
g^{(n)}(k)=\frac{d^{n}}{d\left(k^{\mu}\right)^{n}}\left[\frac{\phi(k)}{\mu k^{\mu-1}}\right] .
$$

It is assumed that the integrand does not possess points of stationary phase in the interval of integration. As before, $\phi(k)$ and its derivatives are seen to appear linearly in $A_{N}$ and $B_{N}$. The arguments of Lemma 4 may be reapplied to yield the following result.

LEMMA 6. If $(\alpha, \beta)$ is a finite interval and $f(k)$ is infinitely differentiable for $0<\alpha \leqq k \leqq \beta$, the asymptotic approximation of $\int_{\alpha}^{\beta} \exp \left(i k^{\mu} r^{\mu}\right) f(k) d k$ to $o\left(r^{-\mu N}\right)$ contains terms evaluated at $\alpha$ which are those terms to $o\left(r^{-\mu N}\right)$ in the expansion 


$$
\sum_{n=0}^{\infty} \frac{\alpha^{n} f^{(n)}(\alpha)}{n !} \int_{\alpha}^{\beta} \exp \left(i k^{\mu} r^{\mu}\right)\left(\frac{k}{\alpha}-1\right)^{n} \eta(k) d k .
$$

For Theorems 1-3, use has been made of the asymptotic expansions of integrals of Bessel functions. A more general formulation may be obtained for functions $F(k r)$ which are continuous and integrable for $0 \leqq k \leqq x<\infty$ and which possess an asymptotic expansion of the form

$$
F(k r)=\sum_{m=0}^{\infty} a_{m}(k r)^{-m+\nu} \exp \left(i k^{\mu} r^{\mu}\right), \quad \mu \geqq 1 .
$$

The integral $T(x r, n)$ may be written as

$$
\begin{aligned}
T(x r, n) & =\int_{0}^{x} F(k r) k^{n} d k \\
& =T(\alpha r, n)+\sum_{m=0}^{N^{\prime}} a_{m} r^{-m+\nu} \int_{\alpha}^{x} k^{-m+n+\nu} \exp \left(i k^{\mu} r^{\mu}\right) d k+o\left(r^{-N^{\prime}+\nu}\right) \\
& =T(\alpha r, n)-T_{N}(\alpha r, n)+T_{N}(x r, n)+o\left(r^{-\mu N+\nu}\right)
\end{aligned}
$$

where

$$
\begin{aligned}
& T_{N}(x r, n)=\sum_{m=0}^{N^{\prime}} a_{m} r^{-m+\nu} B_{N^{\prime}-m, m}\left(r^{\mu}\right), \\
& T_{N}(\alpha r, n)=\sum_{m=0}^{N^{\prime}} a_{m} r^{-m+\nu} A_{N^{\prime}-m, m}\left(r^{\mu}\right)
\end{aligned}
$$

with $B_{p, m}$ and $A_{p, m}$ defined for integer $p$ as the $B_{p}$ and $A_{p}$ of (6.3) and (6.4) with $\phi(k)=$ $k^{-m+n+v}$ and where $N^{\prime}$ is an integer greater than $\mu N$.

From (6.9), it follows that $T(x r, n)$ may be expressed in the form

$$
T(x r, n)=S(n, r)+T_{N}(x r, n)+o\left(r^{-\mu \cdot N+\nu}\right),
$$

where $S$ does not depend on $x$. It is to be noted that the terms of $T_{N}$ possess a factor $\exp \left(i r^{\mu} x^{\mu}\right)$. This result is a generalization of (5.9) and is used in the proof of the following theorem.

THEOREM 4. The asymptotic approximation to $o\left(r^{-N \mu+\nu}\right)$ of the integral $\int_{0}^{\infty} F(k r) f(k) d k$ may be expressed in the form

$$
\int_{0}^{\infty} F(k r) f(k) d k=\sum_{n=0}^{M} \frac{f^{(n)}(0)}{n !} S(n, r)+o\left(r^{-\mu N+\nu}\right),
$$

where $M$ is taken sufficiently large to include all terms in $S(n, r)$ to $o\left(r^{-\mu+\nu}\right)$ and where $S(n, r)$ is defined by

$$
\int_{0}^{\infty} F(k r) k^{n} d k=S(n, r)+T_{N}(x r, n)+o\left(r^{-\mu N+\nu}\right) \text { for } x>0,
$$

\section{provided that}

(1) $F(k r)$ is continuous and integrable in the range $0 \leqq k \leqq x<\infty$ and possesses an asymptotic expansion as $k r \rightarrow \infty$ of the form $F(k r)=\exp \left(i k^{\mu} r^{\mu}\right)(k r)^{\nu} \sum_{i=0}^{\infty} a_{i}(k r)^{-i}$ with $\mu \geqq 1$; 
(2) $f(k)$ is regular analytic in a region of the complex $k$-plane which includes an interval $-\epsilon_{1} \leqq k \leqq \beta+\epsilon_{2}$, for $\epsilon_{1}, \epsilon_{2}$ and $\beta>0$;

(3) $f(k)$ is $N$ times continuously differentiable for $k>0$;

(4) as $r \rightarrow \infty$, $\exp \left( \pm i k^{\mu} r^{\mu}\right) f(k)$ do not possess points of stationary phase for $k>\beta / 2$;

(5) $\left[k^{v} f(k)\right]^{(n)} \rightarrow 0$ as $k \rightarrow \infty$ for $n=0,1,2, \cdots, N-1$; and

(6) $\left[k^{\nu} f(k)\right]^{[N]}$ is integrable over $(\beta, \infty)$.

The proof follows the same line of reasoning as in the previous theorems. The asymptotic approximation of the integral of $F(k r) f(k)$ over $(0, \beta)$ may be expressed as the sum of terms in $f^{(n)}(0) T(\alpha r, n)$ with the terms to $o\left(r^{-\mu+\gamma}\right)$ in the expansion given in Lemma 6 and any terms at $\beta$. The terms at $\beta$ for $(\alpha, \beta)$ cancel the terms for $(\beta, \infty)$ to the order prescribed. The terms remaining are those terms to $o\left(r^{-\mu N+\eta}\right)$ from the expansion

$$
\begin{aligned}
\sum_{n=0}^{\infty} \frac{f^{(n)}(0)}{n !} & \int_{0}^{\beta} F(k r) k^{n} H(k) d k \\
= & \sum_{n=0}^{\infty} \frac{f^{(n)}(0)}{n !} \int_{0}^{\beta} H(k) \frac{d}{d k} T(k r, n) d k \\
& =\sum_{n=0}^{\infty} \frac{f^{(n)}(0)}{n !}\left[S(n, r)-\int_{\alpha}^{\beta} T_{N}(k r, n) \frac{d}{d k} H(k) d k\right]+o\left(r^{-\mu N+\nu}\right),
\end{aligned}
$$

where $T_{N}$ is defined by (6.10), and $H(k)$ is the neutralizer function previously defined. Because of the factor $\exp \left(i k^{\mu} r^{\mu}\right)$ in $T_{N}$, the integral over $(\alpha, \beta)$ vanishes to any prescribed order of $r$ as can be shown through application of Theorem $C$. The result given by (6.13) follows immediately. Q.E.D.

For the purposes of computation, it is worthwhile to note that, as $r \rightarrow \infty$,

$$
\int_{0}^{\infty} e^{-\lambda k} F(k r) d k \sim \sum_{n=0}^{\infty}(-\lambda)^{n} S(n, r) / n ! .
$$

7. Discussion. As examples of applications of Theorem 4, consider the sine and cosine integrals $\operatorname{si}(x)$ and $\operatorname{ci}(x)$. The required properties and results can be obtained from [5]. Since $\operatorname{ci}(x) \sim p(x) \sin x-q(x) \cos x$ where

$$
p(x) \sim x^{-1}\left(1-2 ! x^{-2}+4 ! x^{-4}-\cdots\right)
$$

and

$$
q(x) \sim x^{-2}\left(1-3 ! x^{-3}+5 ! x^{-5}-\cdots\right),
$$

condition (1) of Theorem 4 is satisfied with $\mu=1$ and $\nu=-1$.

Now,

$$
\int_{0}^{\infty} e^{-k} \operatorname{ci}(k r) d k=(2 r)^{-1} \sum_{n=0}^{\infty}(-)^{m} r^{1-2 m} / m
$$

so that

$$
\int_{0}^{\infty} \operatorname{ci}(k r) f(k) d k=-\frac{1}{2} \sum_{n=1}^{M} \frac{(-)^{n} f^{(2 n-1)}(0)}{n r^{2 n}}+o\left(r^{-N-1}\right),
$$

where $M$ is such that $\frac{1}{2}(N+1)>M \geqq \frac{1}{2} N$, provided that $f(k)$ satisfies the conditions of the theorem. Similarly, it can be shown that 


$$
\int_{0}^{\infty} \operatorname{si}(k r) f(k) d k \sim-\sum_{n=0}^{M} \frac{(-)^{n} f^{(2 n)}(0)}{(2 n+1) r^{2 n+1}}
$$

As another example consider $\sin k^{2} r^{2}$. It is well known [5] that the Fresnel integral, defined by

$$
S(x)=\left(\frac{2}{\pi}\right)^{1 / 2} \int_{0}^{x} \sin u^{2} d u,
$$

has an asymptotic expansion of the form

$$
S(x) \sim \frac{1}{2}+g(x) \cos x^{2}+h(x) \sin x^{2},
$$

where $g$ and $h$ possess asymptotic power series expansions in $x^{-1}$. The Laplace transform of $\sin k^{2} r^{2}$ yields the expansion

$$
\begin{aligned}
\int_{0}^{\infty} e^{-u} \sin u^{2} r^{2} d u= & \left(\frac{\pi}{2}\right)^{1 / 2} \frac{1}{2 r}\left[\cos \frac{1}{4 r^{2}}+\sin \frac{1}{4 r^{2}}\right] \\
& -\frac{1}{2 r^{2}}\left[\sum_{n=0}^{\infty} \frac{(-)^{n} r^{-4 n} 2^{-2 n}}{1 \cdot 3 \cdots(4 n+1)}\right]
\end{aligned}
$$

Hence, it may be concluded that

$$
\begin{aligned}
\int_{0}^{\infty} f(k) \sin k^{2} r^{2} d k \sim & \left(\frac{\pi}{2}\right)^{1 / 2} \frac{1}{2 r}\left[f(0)+\frac{f^{\prime \prime}(0)}{4 r^{2}}-\frac{f^{(\mathrm{iv})}(0)}{32 r^{4}}+\cdots\right] \\
& +\frac{1}{2 r}\left[\frac{f^{\prime}(0)}{r}-\frac{f^{(v)}(0)}{60 r^{5}} \cdots\right]
\end{aligned}
$$

which may be verified easily for the integrals

$$
\int_{0}^{\infty} J_{0}(b k) \sin k^{2} r^{2} d k=-\frac{\pi^{1 / 2}}{2 r} \sin \left(\frac{b^{2}}{8 r^{2}}-\frac{\pi}{4}\right) J_{0}\left(\frac{b^{2}}{8 r^{2}}\right)
$$

and

$$
\int_{0}^{\infty} J_{1}(b k) \sin k^{2} r^{2} d k=\frac{1}{b} \sin \frac{b^{2}}{4 r^{2}}
$$

An interesting facet of these expansions is that the function $\exp \left(i b k-i k^{2} r^{2}\right)$ possesses a stationary point at $k=b / 2 r^{2}$. As $r \rightarrow \infty$, this point moves arbitrarily close to the origin. The theorem does not apply to the integral

$$
\int_{0}^{\infty} \operatorname{si}\left(b^{2} k^{2}\right) J_{1}(k r) d k=-\frac{1}{r}\left[\operatorname{si}\left(\frac{r^{2}}{4 b^{2}}\right)+\frac{\pi}{2}\right] .
$$

An application of (1.2) yields only $\pi / 2 r$. It appears that the other term arises because of the stationary point $k=r / 2 b^{2}$ which, as $r$ tends to infinity, does not remain confined to an interval $0<k \leqq \beta / 2<\infty$ as required by condition (5).

Further examples of the application of Theorem 4 are provided by the Fresnel integrals [5] defined by (7.4) and the following:

$$
C(x)=\left(\frac{2}{\pi}\right)^{1 / 2} \int_{0}^{x} \cos u^{2} d u .
$$


It can be shown readily that $\frac{1}{2}-S(k r)$ and $\frac{1}{2}-C(k r)$ satisfy requirement (1) of Theorem 4. From their Laplace transforms, one obtains the following results for suitable $f(k)$ :

$$
\begin{aligned}
\int_{0}^{\infty}\left\{\frac{1}{2}-S(k r)\right\} f(k) d k= & \frac{1}{(2 \pi)^{1 / 2}} \frac{f(0)}{r}+\frac{1}{8} \frac{f^{\prime}(0)}{r^{2}}-\frac{1}{64} \frac{f^{\prime \prime \prime}(0)}{r^{4}} \\
& -\left(\frac{2}{\pi}\right)^{1 / 2} \frac{1}{120} \frac{f^{(i \mathrm{i})}(0)}{r^{5}}-\frac{1}{768} \frac{f^{(v)}(0)}{r^{6}}+o\left(r^{-8}\right), \\
\int_{0}^{\infty}\left\{\frac{1}{2}-C(k r)\right\} f(k) d k= & -\frac{1}{8} \frac{f^{\prime}(0)}{r^{2}}-\left(\frac{2}{\pi}\right)^{1 / 2} \frac{1}{12} \frac{f^{\prime \prime}(0)}{r^{3}}-\frac{1}{64} \frac{f^{\prime \prime \prime}(0)}{r^{4}} \\
& +\frac{1}{768} \frac{f^{(v)}(0)}{r^{6}}+\left(\frac{2}{\pi}\right)^{1 / 2} \frac{1}{1680} \frac{f^{(\mathrm{vi})}(0)}{r^{7}}+o\left(r^{-8}\right) .
\end{aligned}
$$

Defence Research Establishment Pacific

Victoria, B. C., Canada

1. H. F. Winlis, "A formula for expanding an integral as a series," Philos. Mag., v. (7) 39, 1948, pp. 455-459. MR 10, 22.

2. A. ERDÉLYI, "Asymptotic representations of Fourier integrals and the method of stationary phase," J. Soc. Indust. Appl. Math., v. 3, 1955, pp. 17-27. MR 17, 29.

3. G. N. Watson, Theory of Bessel Functions, 2nd ed., Cambridge Univ. Press, Cambridge; Macmillan, New York, 1944. MR 6, 64.

4. Y. L. LUKE, Integrals of Bessel Functions, McGraw-Hill, New York, 1962. MR 25 \#5198.

5. I. S. GRadštrǏn \& I. M. RyžIK, Tables of Integrals, Series and Products, 4th ed., Fitzmatgiz, Moscow, 1963; English transl., Academic Press, New York, 1965. MR 28 \#5198; MR 33 \#5952. 